\title{
Effects of the COVID-19 Pandemic and How We Adapted at the University of Missouri
}

\author{
Richard J. Barohn, MD \\ University of Missouri School of Medicine \\ Executive Vice Chancellor for Health Affairs \\ Executive Director, NextGen Precision Health initiative
}

I joined the University of Missouri (MU) in May 2020, during the early phases of the COVID-19 pandemic. My leadership administrative roles at MU are in the School Lof Medicine, MU Health Care, and in the NextGen Precision Health initiative. My role as the Executive Vice Chancellor of Health Affairs at the University of Missouri allows me to develop and implement a comprehensive strategy that affirms MU Health Care as a nationally recognized leader in patient care and to continue the MU School of Medicine's legacy of education and scholarly excellence.

How We Adapted to COVID-19 on a Multi-Center Clinical Trial

As a clinician researcher, I moved an investigator-initiated, federally funded grant to MU from the University of Kansas and remain a primary investigator. The study, "The ALS Memantine Trial Study (TAME)", ${ }^{1}$ is a phase II study of the drug memantine for patients with amyotrophic lateral sclerosis (ALS), also known as Lou Gehrig's disease. It is an R01 funded study by FDA Office of Orphan Products Development. TAME is a multi-center, clinical trial of a drug that is currently FDA approved for the treatment of dementia in Alzheimer's disease. Our research team speculated that the drug may also be effective for ALS. As a phase II trial, our main aims were to establish that the drug is safe in high doses among ALS patients and to explore the effect of the drug on blood biomarkers for ALS. We were also trying to detect if the drug could slow the progression of ALS or cause an improvement of the neuropsychiatric symptoms involving cognition changes that are characteristic of ALS. This was not meant to be an efficacy study. The goal was to enroll 90 patients with ALS at 13 centers around the country (Table 1). The trial is a randomized control trial with a 2:1 randomization so that 60 patients would get the active drug and 30 would receive the placebo.

The COVID-19 pandemic had a significant impact on our approach and required changes to how we operationalized this study. On March 18, 2020, in the early phase of the COVID-19 pandemic, all academic health centers around the country announced that protocols involving research patients enrolled in trials

Table 1. TAME Site Table

\begin{tabular}{|c|c|}
\hline Site \# & Name \\
\hline 1 & University of Kansas Medical Center \\
\hline 2 & Nerve \& Muscle Center of Texas \\
\hline 3 & University of Missouri \\
\hline 4 & University of Washington \\
\hline 5 & University of Kentucky \\
\hline 6 & UC - Irvine \\
\hline 7 & University of Kansas - Wichita \\
\hline 8 & Cox Health - Springfield \\
\hline 9 & University of Florida \\
\hline 10 & Providence Neurological \\
\hline 11 & Penn State Hershey Medical Center \\
\hline 12 & Austin Neuromuscular Center \\
\hline 13 & Prolth \\
\hline
\end{tabular}


must be suspended because outpatient operations were shutting down. How did we adapt?

We immediately met with the study's steering committee, investigators, and coordinators at each study site to determine their ability to continue work on the project. We discovered that all sites were affected in somewhat different ways depending on the local policies at each university, but all had to suspend seeing ALS patients for research visits. We had to come up with an adapted protocol so that we could enroll new patients and continue seeing enrolled patients remotely using phone calls and video visits. We created an alternate set of inclusion and exclusion criteria that allowed for the participant to be enrolled partially or completely remotely. We used recently collected clinical data from the previous clinic visit to capture items that were needed for clinical enrollment, such as physical examination and vital capacity. We sent the modified protocol to the IRB at KU Medical Center. Because this was a federally funded multi-center trial, we were operating under the new rules that mandate the use of a central IRB. This turned out to be an advantage in this situation. Once the KU Medical Center IRB approved the modified protocol, we were able to quickly get the protocol approved through an administrative approach at all the other sites, which did not require additional IRB approval at those sites. Simultaneously, we sent an updated protocol to the FDA funding agency and FDA regulatory agency. We established a mechanism to send patients their study drug or placebo drug directly to their homes. Research coordinators provided monthly phone calls or video visits with patients and their families. We were able to maintain the collection of study data, such as patient ALS functional rating scale, adverse events, and medication counts remotely.

Figure 1 shows our study enrollment from the beginning of the study in November 2018 to the end of enrollment in September 2020. Amazingly, due to the changes we made in the protocol and to a commitment by the investigators, coordinators, and patients that we would not slow the study because of the COVID-19 pandemic, we actually increased the pace of study enrollment in the closing five

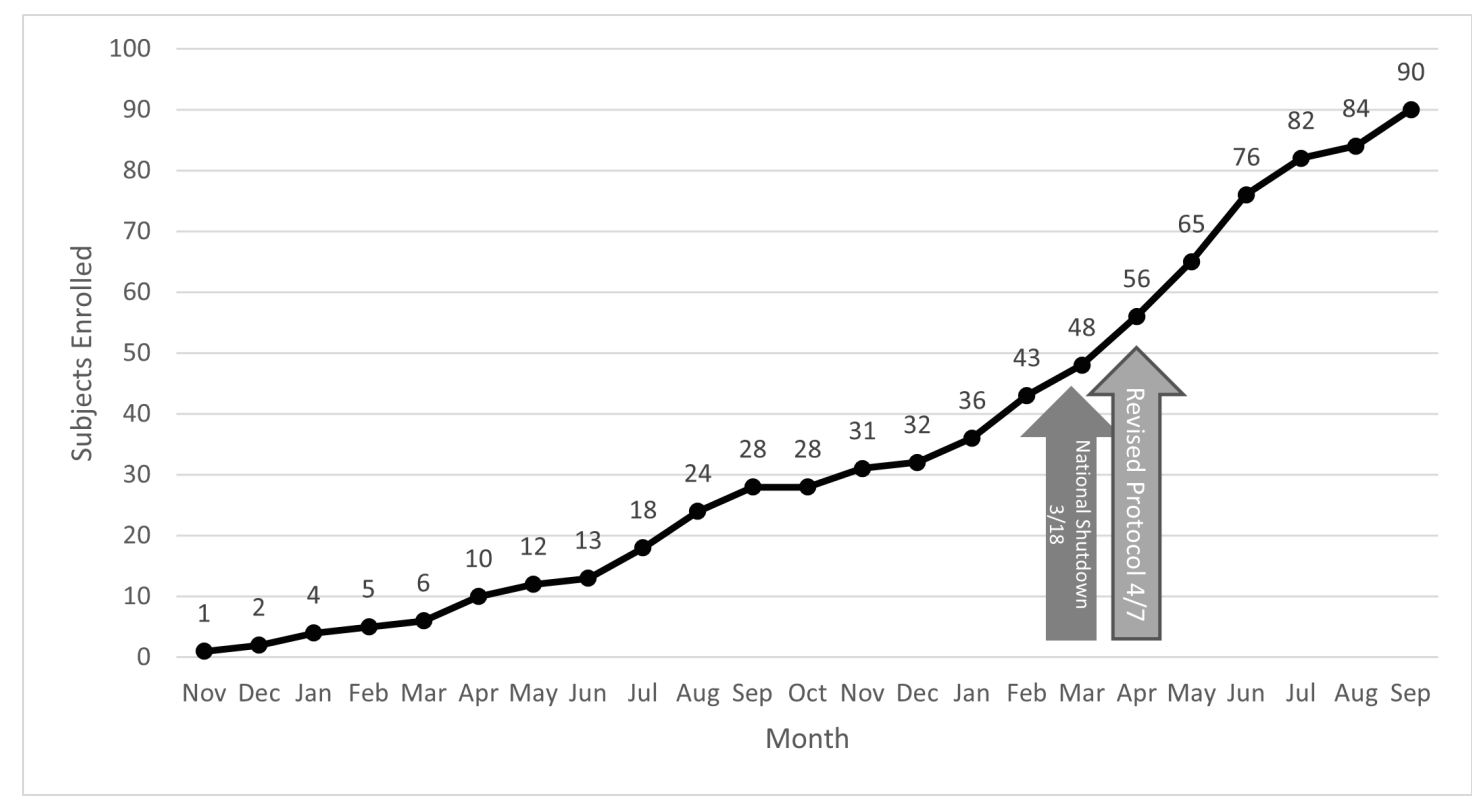

Figure 1. The number of subjects enrolled in TAME study from November 2018 through September 2020. 
months of the study. Prior to using the new protocol, we enrolled 56 patients from November 2018 to April 2020; from April 2020 to September 2020, we enrolled 34 patients. We learned several lessons from this experience. We were quickly able to adapt the protocol, get buy-in from our investigators, get timely administrative review, and stay on target for completing enrollment. We found that using the central IRB mechanism with administrative approvals by each local IRB worked very well in making quick changes to the protocol that could be adopted at all sites. There is no doubt that many of the lessons learned under pressure from the COVID-19 pandemic will be useful in designing and conducting future clinical trials, irrespective of the pandemic situation.

Global Impact of the COVID-19 Pandemic on MU Research

Despite this positive outcome regarding the TAME study, overall research activity drastically decreased in 2020 . Essentially, all laboratory studies were put on hold. By May 20, 2020, the University of Missouri began slowly initiat- ing a research restart plan called "Show Me Renewal," and we began re-opening some laboratories and outpatient clinical research operations.

Because of COVID-19, MU investigators were able to become involved in nine COVID-19 clinical trials. In addition, our investigators were involved in 45 additional COVID-19-based research studies that included natural history studies, laboratory-tested studies, and non-trial clinical protocols. One of the problems we encountered with the drugs for the COVID-19 clinical trials was, at the time, not having enough COVID-19 patients hospitalized at MU Health Care to successfully recruit in each trial, particularly during the early phases of the pandemic. That did change in the fall and winter months of 2020 when our COVID-19 patient numbers increased and our maximum number of patients in the hospital was just under 100 during our highest daily census.

Another dimension of the COVID-19 pandemic has been its impact on finance. We saw an impact on our research expenditures during fiscal year (FY) 2021. From

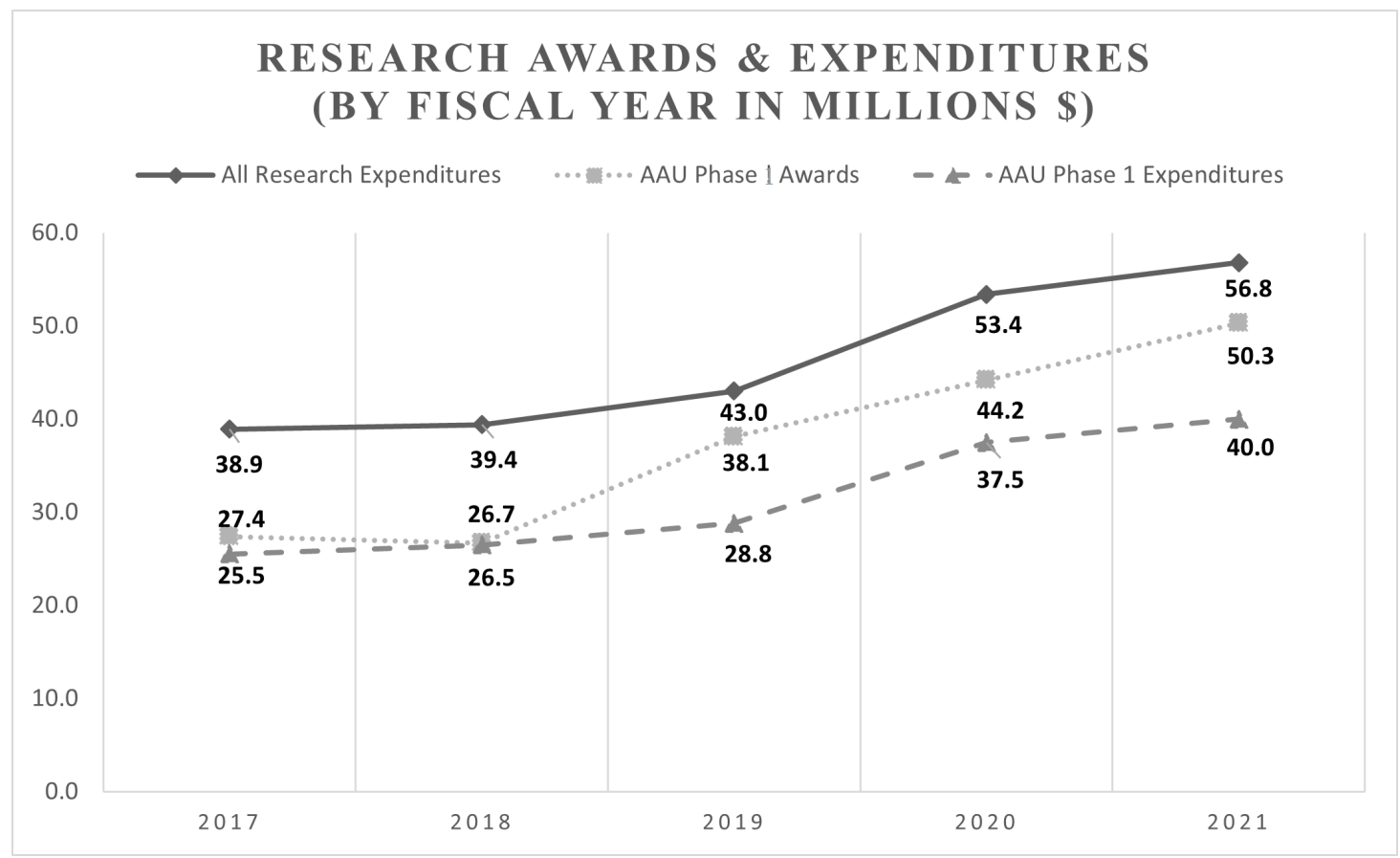

Figure 2. University of Missouri research metrics during COVID-19. 
FY19 to FY20, our research expenditures increased by $9.7 \%$. However, from FY20 to FY21, while our research expenditures did increase, the increase was only $2.6 \%$ (See Fig. 2, previous page). We believe this drop in our research expenditure growth and research award dollars was due to the COVID-19 pandemic. We have also seen substantial growth in AAU Phase 1 Awards, as noted in Figure 2, which is a leading indicator for growth in AAU Phase 1 expenditures (represented by the dashed line).

As a result of the COVID-19 pandemic, faculty began writing scholarly articles. There were 154 COVID-19 articles written by MU faculty over the last year, and these articles have received over 2,000 citations. Some of the projects involving COVID-19 research were quite cleverly designed. One example is the study "Mandated Societal Lockdown and Road Traffic Accidents" by Adnan Qureshi, MD, and colleagues. ${ }^{2}$ The authors used the statewide Traffic Accident Records System maintained by Missouri State Highway Patrol to determine if the mandated stay-at-home policies led to a reduction in road traffic accidents or fatalities.

The University of Missouri Health Care Response to COVID-19

On March 13, 2020, the University of Missouri hospital (MUHC) initiated an Incident Command leadership structure, as did most academic health centers and hospitals around the country, and held the first meeting. Our first COVID-19 case was announced on March 17, 2020. The Incident Command infrastructure was led by Stevan Whitt, MD, and Mary Beck, DNP, RN. The Incident Command team consisted of the following leaders: Incident Commander, Ambulatory Section Chief, Logistics Section Chief, Infection Control Section Chief, Planning Section Chief, Vaccination Team, Liaison Officer, Public Information Officer, Finance Section Chief, and Human Resources Section
Chief. The Incident Command team initially met twice a day, seven days a week. Initially, I joined these sessions virtually and found them to be quite beneficial as I was able to meet new colleagues at MU Health Care prior to being in Columbia to join in person. I was able to gain an awareness of their leadership roles and skills in a way that would not have happened during "normal times."

As previously mentioned, our highest daily census of hospitalized COVID-19 patients occurred in December 2020 and January 2021 (see Fig. 3). Figure 3 displays the daily COVID-19 inpatient census from the beginning of the pandemic to the submission of this article. By April 2021, our numbers dwindled, with some days showing we had zero to five COVID-19-positive patients in the hospital. Due to the surge of infections during the summer of 2021, case numbers increased. At the time this article was written (August 2021), our average daily census of COVID-19-positive hospitalized patients is 70 to 80 .

An outcome of the COVID-19 pandemic was a dramatic increase in the use of telemedicine. Because patients could not come into the clinics, clinicians rapidly figured out how to see these patients via telephone or video. Our weekly telemedicine visits went from zero to 4,000 per week. However, with the waning of the pandemic, the number of telehealth visits has decreased dramatically (Fig. 4). Maintaining momentum in the effective use of telemedicine is a missed opportunity that is unfortunately occurring around the country. ${ }^{3}$ It would be valuable to find ways to convince payers, doctors, nurses, and patients to continue to take advantage of the benefits of telemedicine in a post-pandemic environment.

There were other challenges and opportunities that resulted from the COVID-19 pandemic. Our hospital became full of very sick COVID-19-positive patients, and our team of providers and 


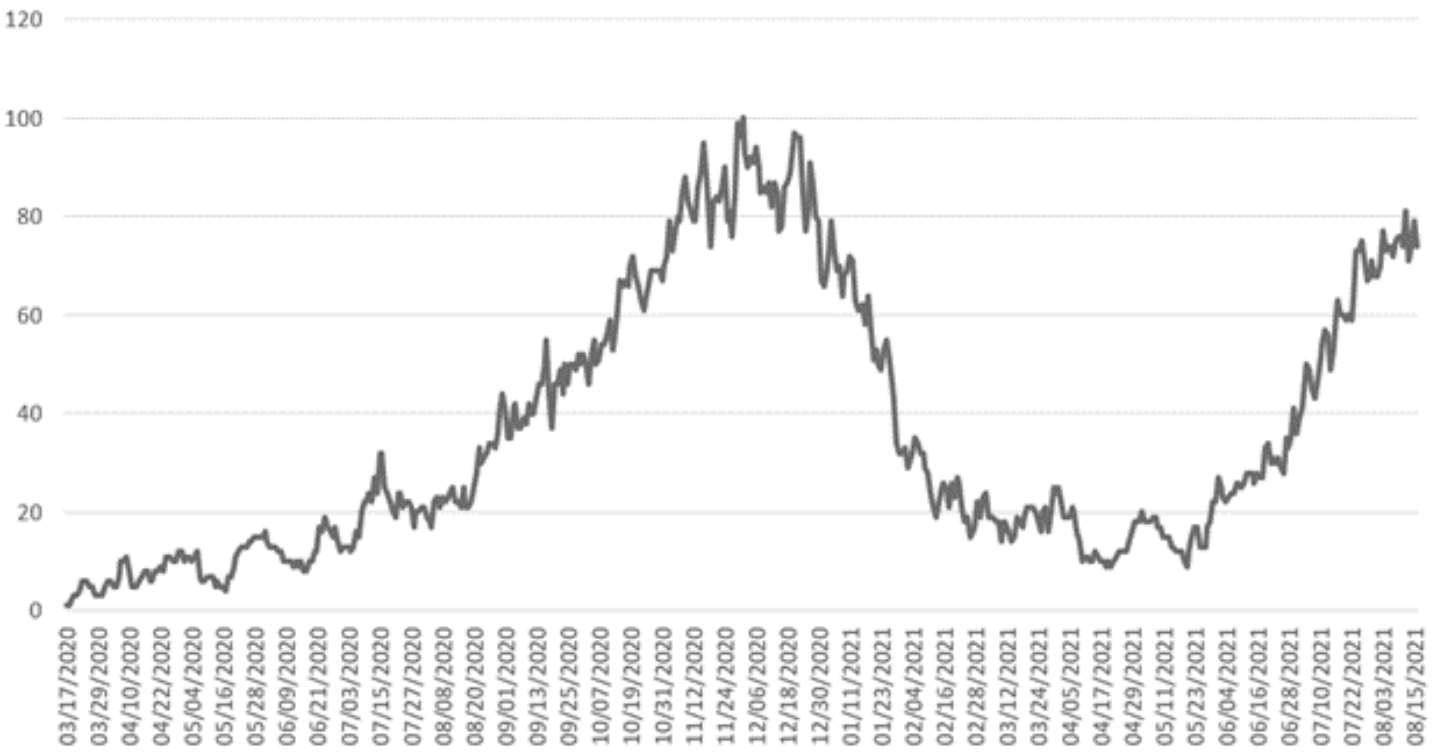

Figure 3. Comparing the COVID-19 inpatient census to the number of telehealth visits that occurred between March 2020 through August 2021.

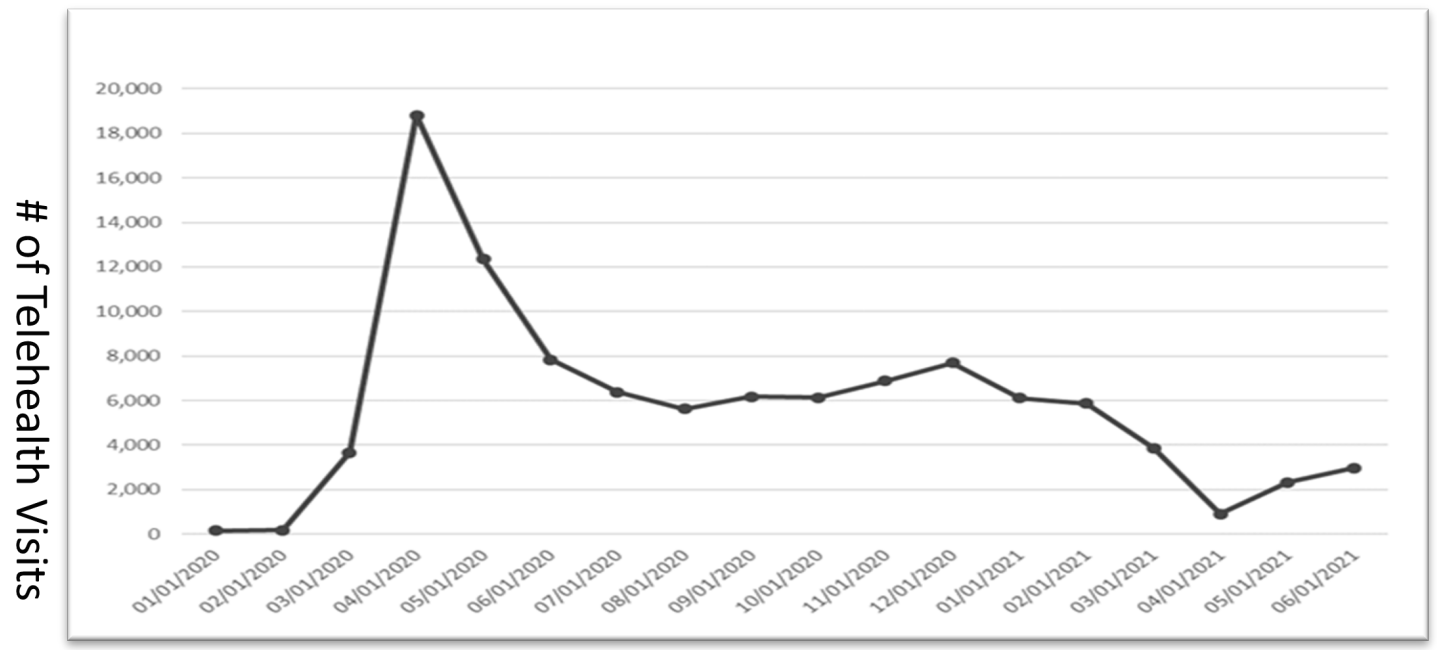

Figure 4. Changes in the volume of telemedicine visits between January 2020 and June 2021.

staff had to use all their skills to adapt to this new infectious challenge. It was a very stressful situation for our doctors, nurses, and other staff members. We witnessed significant burnout as the stress built up on these health care providers. Therefore, our health system leadership team and wellness program adapted to the situation by sending out daily messages to maintain confidence and morale. Everyone wanted to know what was happening as a result of the COVID-19 pandemic in an environment of rapidly shifting priorities, so hospital leaders set up weekly online town hall meetings to inform faculty, staff, and students of the situation in the hospital, on campus, and within the community. After the summer months of 2020, we decreased town halls to twice a month; then after the winter surge, we decreased the town halls to monthly, and we maintain them on a 
quarterly schedule. This again may be a lost opportunity as many people valued these communication exchanges with leadership.

Quickly getting a vaccination system operational was another challenge. We initially set up vaccination clinics in the basement of the hospital but realized there was a community need for vaccination that was not being met by local clinics or local public health. We shifted the vaccination location to the University's football stadium where we could safely manage and vaccinate several thousand people a day. We contribute to a successful vaccination rate of over $50 \%$ among the citizens in Boone County.

Finally, the COVID-19 pandemic clearly had an impact on MU Health Care's financial position. In FY20, our operating income decreased to $\$ 47.5$ million compared to an operating income in FY19 of $\$ 83.3$ million. This was directly due to the shutdown of the hospital in the early months of the COVID-19 pandemic with the exception of COVID-19 cases and true medical emergencies. The shutdown essentially affected the last quarter of 2020 . Fortunately, there has been a robust rebound in 2021, and we are expecting an end of academic year operating income of over $\$ 90$ million.

The University of Missouri School of Medicine also had to pivot sharply because of the COVID-19 pandemic. We switched to a completely online program for our medical students in March 2020. By May 2020, we began allowing some students back into the clinic and hospital setting under restricted conditions. Currently (August 2021), we essentially are back to a pre-COVID-19 teaching environment for medical students, but that could change if the summer 2021 surge of infections and hospitalizations continue to increase.

On May 13, 2021, we held a COVID-19 recognition ceremony. We had an outdoor gathering for staff, faculty, and students where we shared a number of experiences. This was a time of remembrance and recognition. We had patients who lost loved ones to COVID-19 speak to the group and had survivors involved in the recognition event. This event of course did not bring full closure to the COVID-19 situation but was a very helpful and healing process.

\section{Ongoing Challenges and Opportunities}

As the COVID-19 pandemic continues to present challenges, we are prepared to make adjustments and seek opportunities to overcome obstacles. One of the challenges the University faces is faculty recruitment and relocation. Since the pandemic began, site and travel restrictions have made it difficult to conduct interviews and forced the elimination of site visits for potential new faculty. To address this, we conduct initial interviews via video calls. This solution may be adopted routinely going forward to save time and money on initial interviews. Second, the COVID-19 pandemic crisis has led to a housing shortage nationwide, and incoming faculty are finding it difficult to find homes to buy. A third challenge has been poor statewide vaccination uptake and risks from new variants of COVID-19. Because of this, we reinstated our mask policy in July 2021 across the MU Health Care campus. There is an opportunity to educate and inform the community about the importance of being vaccinated. While overall research has been challenged because of the pandemic, we see an increased opportunity for the NextGen Precision Health initiative at the University of Missouri. Initially consisting of three main research focus areas, the initiative now includes infectious disease research. Our Influenza+ Center includes COVID-19 research and is in the process of hiring additional staff and faculty.

The COVID-19 pandemic continues to have a global impact. Healthcare sys- 
tems, public health, medical education, and clinical practice have responded to the challenge of dealing with a pandemic in innovative and impactful ways. From a system level to an individual level, creative problem solving and meeting the responsibilities to care for patients and communities have been a hallmark of our response. One of the greatest discoveries has been our ability to adapt and move forward to better serve our community.

\section{References}

1. Levine, T., Barohn, R. J., Goyal, N., Pulley, M., Schwasinger-Schmidt, T., Kasarskis, E., Govindarajan, R., Papsdorf, T., Simmons, Z., Shaibani, A., \& Wiss, M. (2019). Therapy in Amyotrophic Lateral Sclerosis (TAME). https://clinicaltrials.gov/ct2/ show/NCT02118727.

2. Qureshi, A. I., Huang, W., Khan, S., Lobanova, I., Siddiq, F., Gomez, C. R., \& Suri, M. F. K. Mandated societal lockdown and road traffic accidents. Accident Analysis Prevention. 2020 Oct; 146:105747. doi: 10.1016/j.aap.2020.105747. Epub 2020 Sep 7. PMID: 32911131; PMCID: PMC7475733. https://pubmed.ncbi.nlm.nih. gov/32911131/

3. Demeke, H. B., Merali, S., Marks, S., Zilversmit Pao, L., Romero, L., Sandhu, P., Clark, H., Clara, A., McDow, K. B., Tindall, E., Campbell, S., Bolton, J., Le, X., Skapik, J. L., Nwaise, I., Rose, M. A., Strona, F. V., Nelson, C., \& Siza, C. (2021). Trends in Use of Telehealth Among Health Centers During the COVID-19 Pandemic - United States, June 26-November 6, 2020. https://www.cdc.gov/mmwr/volumes/70/wr/ pdfs/mm7007a3-H.pdf 